\title{
Intra-arterial Contrasted Cone-beam Computed Tomography Assessment of Vessels Distal from Occluded Site in Acute Ischemic Stroke with Major Vessel Occlusion
}

\author{
Tatsuo Amano, ${ }^{1}$ Masayuki Sato, ${ }^{1}$ Yuji MATSUMARU, ${ }^{1}$ Hideyuki SAKUMA, ${ }^{2}$ \\ Syogo YODA, ${ }^{2}$ and Yusuke HAMADA ${ }^{2}$ \\ ${ }^{1}$ Department of Neuro-Endovascular Therapy, Toranomon Hospital, Tokyo, Japan; \\ ${ }^{2}$ Department of Radiology, Toranomon Hospital, Tokyo, Japan
}

\begin{abstract}
Characterization of vessels distal from occluded site is important when considering endovascular revascularization therapy (EVT) for acute ischemic stroke. The goal of this study was to assess the clinical value of intra-arterial contrasted high-resolution cone-beam computed tomography from the ascending aorta (Ao-CBCT) for visualization of the vessels distal from occluded site. Acute ischemic stroke patients with large vessel occlusion who were to undergo EVT were evaluated. In EVT, digital subtraction angiography (DSA) and Ao-CBCT were performed with local anesthesia. Ao-CBCT images were acquired in a 20-second rotational scan. Contrast medium was injected $(1 \mathrm{~mL} / \mathrm{s}$ for a total of 30 seconds using a 4-Fr catheter and an imaging delay of 10 seconds) from the ascending aorta. We assessed the image quality of Ao-CBCT and compared the visualization of the vessels distal from occluded site among magnetic resonance angiography (MRA), DSA and Ao-CBCT. We analyzed 14 patients (mean age, 66 years; three female patients). Stroke subtypes were cardiogenic $(n=6)$, atherothrombotic $(n=5)$ and others/unknown $(n=3)$. Occluded sites were middle cerebral artery (MCA) M1 $(n=8)$, MCA M2 $(n=2)$, internal carotid artery (ICA) $(n=2)$, MCA M4 $(n=1)$ and basilar artery (BA) $(n=1)$. All obtained Ao-CBCT images successfully characterized the vessels distal from occluded site, and 11 images (79\%) were excellent. In all cases, Ao-CBCT images could depict distal vessels with more detail when compared with MRA and DSA. Ao-CBCT is an efficient method to obtain detailed information regarding vessels distal from occluded site when compared with conventional examination methods.
\end{abstract}

Key words: ischemic stroke, mechanical thrombectomy, cone beam CT, large vessel occlusion, collateral flow

\section{Introduction}

Mechanical thrombectomy results in a high revascularization rate in patients with acute ischemic stroke. ${ }^{1-4)}$ Recently, several trials reported stent retriever thrombectomy improved clinical outcomes compared with medical therapy alone. ${ }^{5-9)}$ Stent retriever is required to advance a microcatheter distal to the thrombus before deployment. Further, it is necessary to predict the vessel diameter and the thrombus length for a suitable selection of device diameter and length. Therefore, it is useful to characterize vessels distal from occluded site. The frequency of procedural complications during endovascular therapy (EVT) for acute ischemic stroke ranges from 3.5 to $16 \%{ }^{1-4)}$ These complications, especially dissection and vessel perforation,

Received December 8, 2016; Accepted February 2, 2017 are considered to occur when the thrombectomy device passes through the occluded site or when the microwire advances through the invisible distal vessels. There, information regarding the distal portion of the occluded site is also important to prevent procedural complications.

Vessels distal form occluded site can be visualized by computed tomography (CT ${ }^{10)}$ or intravenous cone beam CT (IV-CBCT). ${ }^{11)}$ Because the time window for EVT is limited, imaging examinations must be performed as quickly as possible. Moreover, the allowable amount of contrast medium is sometimes limited by the presence of renal dysfunction.

The goal of the present study was to assess the clinical utility of high-resolution CBCT with contrast injected from the ascending aorta (Ao-CBCT) for the visualization of the vessels distal from occluded site. 


\section{Materials and Methods}

\section{Subjects and imaging protocol}

After review of the protocol by our local ethics committee, informed consent was obtained from all patients or their family before EVT. We enrolled consecutive acute ischemic stroke patients with large vessel occlusion who were planned to undergo EVT between October 2012 and July 2013. Using a 1.5-T scanner, all patients were initially assessed by magnetic resonance imaging (MRI) and magnetic resonance angiography (MRA) to evaluate brain ischemia and large vessel occlusion. Intravenous tissue plasminogen activator (tPA) therapy was performed before EVT if it was eligible. Patients with Alberta Stroke Program Early Computed Tomography-diffusion weighted image (ASPECT-DWI) score $>5$ based on initial MRI and large vessel occlusion [internal carotid artery (ICA), middle cerebral artery (MCA) M1/M2, basilar artery (BA) and vertebral artery (VA)] based on initial MRA were eligible for EVT. EVT was performed if neurological improvement was not obtained at 30 min after tPA administration. The Merci retriever (Concentric Medical) and Penumbra system (Penumbra) were used for EVT.

Patients underwent conventional angiography and Ao-CBCT under local anesthesia with propofol and fentanyl. An 8-Fr or 9-Fr catheter sheath was introduced via the femoral artery. Diagnostic angiography and EVT were performed with an 8-Fr or 9-Fr guiding catheter and a 4Fr JB2 catheter coaxial system.

Ao-CBCT images were acquired after non-diluted contrast injection $(1.0 \mathrm{ml} / \mathrm{s}$ for a total amount of 30 $\mathrm{ml}$ ) from the ascending aorta with a contrast injector. The delay time for the cone-beam CT (Allura Xper FD 20/20; Philips Healthcare) acquisition was 10 seconds with targeting of the occluded vessel into the center of rotation. Collected 2-dimensional raw data were automatically sent to the dedicated workstation (Xtravision; Philips Healthcare) and reconstructed to the 3-dimensional image within a few minutes. After Ao-CBCT, a guiding catheter was advanced into target vessel. If the operator considered that more vessel information was necessary, additional digital subtraction angiography (DSA) was performed in other vessels.

\section{Assessment and analysis of images}

We assessed the distal portions of the occluded site using MRA, DSA and Ao-CBCT images. Evaluation of vessels distal from occluded site was based on retrograde contrast opacification of vessels within the occluded vessel territory. We used the following scoring system: ${ }^{12)}$

1. A score of 1 was assigned if collaterals reconstituted the distal portion of the occluded vessel segment (i.e., if there was M1 segment occlusion, the M1 segment distal to the occlusion reconstituted).

2. A score of 2 was assigned if collaterals reconstituted vessels in the proximal portion of the segment adjacent to the occluded vessel (i.e., if there was M1 segment occlusion with reconstitution to the proximal M2 vessel segments).

3. A score of 3 was assigned if collaterals reconstituted vessels in the distal portion of the segment adjacent to the occluded vessel (i.e., if there was M1 segment occlusion with reconstitution to the distal portion of the M2 vessel segments).

4. A score of 4 was assigned if collaterals reconstituted vessels two segments distal to the occluded vessel (i.e., if there was M1 segment occlusion with reconstitution up to the M3 segment branches).

5. A score of 5 was assigned if there was little or no significant reconstitution of the territory of the occluded vessel.

For image quality evaluation of Ao-CBCT, we defined grading criteria as three categories based on the boundary between vessels and surrounding anatomical structures: 1) good: more than 50\% clear boundary between vessels and other anatomical structures; 2) fair: less than $50 \%$ clear boundary between vessels and other anatomical structures; or 3) poor: difficult to identify the boundary between vessels and other anatomical structures.

\section{Results}

\section{Patient characteristics}

We evaluated 14 patients (mean age, 66 years; three female patients). The mean baseline National Institutes of Health Stroke Scale (NIHSS) score was 17. Stroke subtypes classified by the trial of Org 10172 in Acute Stroke Treatment (TOAST) criteria were cardiogenic $(n=6)$, large-artery atherosclerosis $(n=5)$ and others/unknown $(n=3)$. Occluded sites identified by initial MRA were MCA M1 $(n=8)$, MCA M2 $(n=2)$, ICA $(n=2)$, MCA M4 $(n=1)$ and BA $(n=1)$. Intravenous tPA therapy was performed in seven patients. The mean time from MRI examination to groin puncture was 117 min (Table 1).

\section{Ao-CBCT versus MRA/DSA}

In all cases, the distal vessels from occluded site could be assessed on Ao-CBCT images. Good image quality of Ao-CBCT was acquired in 11 of 14 cases $(79 \%)$. Because of motion artifact, fair image quality was acquired in three of 14 cases (21\%) (Table 2).

Visualization scores of the distal vessels from occluded site were $1(n=1), 2(n=1)$ and $5(n=12)$ on MRA; $1(n=1), 2(n=4)$ and $5(n=4)$ on DSA; and $1(n=10), 2(n=3)$ and $3(n=1)$ on Ao-CBCT 
Table 1 Baseline characteristics and treatment

\begin{tabular}{|c|c|c|c|c|c|c|c|}
\hline No & Age & Sex & NIHSSscore & Etiology & IV-tPA & Endovascular therapy & $\begin{array}{l}\text { TICI grade } \\
\text { post EVT }\end{array}$ \\
\hline 1 & 38 & $\mathrm{M}$ & 16 & others & - & Penumbra & $2 a$ \\
\hline 2 & 64 & M & 24 & cardioembolic & + & Penumbra & $2 \mathrm{~b}$ \\
\hline 3 & 37 & M & 21 & cryptogenic & + & Penumbra & $2 \mathrm{~b}$ \\
\hline 4 & 81 & $\mathrm{~F}$ & 18 & cardioembolic & + & Merci & $2 \mathrm{a}$ \\
\hline 5 & 78 & M & 15 & ATBI (A to A) & + & CAS, Penumbra & $2 b$ \\
\hline 6 & 70 & M & 20 & ATBI (A to A) & + & CAS, Penumbra & $2 \mathrm{~b}$ \\
\hline 7 & 68 & M & 19 & $\begin{array}{l}\text { ATBI (Atherosclerotic } \\
\text { occlusion) }\end{array}$ & - & Balloon PTA & $2 a$ \\
\hline 8 & 80 & M & 6 & cardioembolic & - & none & - \\
\hline 9 & 65 & $\mathrm{~F}$ & 12 & cardioembolic & - & none & - \\
\hline 10 & 72 & $\mathrm{M}$ & 12 & ATBI (A to A) & - & CAS & 3 \\
\hline 11 & 77 & $\mathrm{M}$ & 26 & others & - & Penumbra & $2 \mathrm{~b}$ \\
\hline 12 & 64 & $\mathrm{M}$ & 21 & ATBI(A to A) & + & CAS, Penumbra & $2 b$ \\
\hline 13 & 71 & $\mathrm{~F}$ & 22 & cardioembolic & - & Penumbra & $2 b$ \\
\hline 14 & 64 & M & 9 & cardioembolic & + & Penumbra & $2 b$ \\
\hline
\end{tabular}

ATBI: indicates atherothrombotic brain infarction, CAS: carotid artery stenting, EVT: endovascular therapy, IV-tPA: intravenous tissue plasminogen activator, NIHSSscore: National Institute of Health stroke scale score, PTA: percutaneous transluminal angioplasty, TICI: thrombolysis in cerebral infarction.

assessment. In all 14 cases, more detailed visualization of the vessels distal from occlude site was possible by Ao-CBCT when compared to MRA or DSA.

\section{Case presentation}

Case 1 (Patient No. 5): A 78-year-old male with a past history of neck radiation therapy for esophageal cancer presented with right ICA stenosis and right MCA M1 occlusion as defined by carotid artery ultrasonography and MRA (NIHSS score 15) (Fig. 1). He was treated with iv-tPA $(0.6 \mathrm{mg} / \mathrm{kg})$ at other hospital but symptoms did not improve. He was referred for EVT and transferred to our hospital. Groin puncture was performed at $128 \mathrm{~min}$ after MRA assessment. Under local anesthesia, Ao-CBCT was performed before conventional angiography. DSA showed right MCA occlusion and leptomeningeal collateral flow (MCA M2 distal segment). Ao-CBCT also showed right MCA occlusion. Only Ao-CBCT revealed MCA M2 bifurcation and distal vessels. After EVT with the Penumbra system followed by carotid artery stenting, thrombolysis in cerebral infarction (TICI) $2 \mathrm{~b}$ recanalization was acquired.

Case 2 (Patient No. 8): An 80-year-old male with atrial fibrillation presented with right MCA occlusion defined by MRA (NIHSS score 6) (Fig. 2). Iv-tPA therapy was contraindicated, because he presented to our hospital at 3.5 hours after stroke onset. To assess the indication for EVT, angiographic assessment was performed. Groin puncture was performed at 325 min after MRA assessment. Under local anesthesia, Ao-CBCT was performed before conventional angiography. DSA showed right MCA proximal occlusion and slight retrograde filling of the distal MCA branches (MCA M2 segment). Ao-CBCT revealed the occluded MCA M1 segment clearly with opacification of the distal MCA territory. We did not perform EVT, because his symptoms were improving.

\section{Discussion}

In this study, Ao-CBCT could visualize distal vessels of the occluded site in all cases. In 11 of 14 cases, good imaging quality was obtained via Ao-CBCT. Compared with conventional imaging examination, Ao-CBCT yielded more detailed vessel information. When using stent retriever devices, the device size must correspond to the vessel diameter. Furthermore, when using stent retrievers, operators have to select the device length that corresponds to thrombus length. With Ao-CBCT imaging, thrombus length as well as vessel diameter distal from occluded site can be estimated before device selection without time delay. Moreover, suitable device selection from Ao-CBCT imaging may reduce procedural complications, such as dissection or vessel perforations 
Table 2 Occlusion site and evaluation of MRA, DSA and Ao-CBCT

\begin{tabular}{rlcccc}
\hline & & \multicolumn{3}{c}{$\begin{array}{c}\text { collateral information } \\
\text { (grade) }\end{array}$} & $\begin{array}{c}\text { graphic } \\
\text { quality }\end{array}$ \\
\cline { 3 - 5 } & & occlusion site & DSA & Ao-CBCT & \\
\hline 1 & MCA M1-D & 5 & 3 & 2 & good \\
2 & BA & 2 & NA & 1 & fair \\
3 & MCA M2 & 5 & 3 & 2 & fair \\
4 & MCA M1-D & 5 & 2 & 1 & good \\
5 & MCA M1-D & 5 & 3 & 1 & good \\
6 & ICA-T & 5 & 4 & 3 & good \\
7 & ICA-D & 1 & 1 & 1 & good \\
8 & MCA M1-P & 5 & 2 & 1 & good \\
9 & MCA M4 & 5 & 5 & 1 & good \\
10 & MCA M1-D & 5 & 4 & 1 & good \\
11 & MCA M2 & 5 & 3 & 1 & good \\
12 & ICA-P+MCA & 5 & 5 & 2 & fair \\
& M1-P & & & & good \\
13 & MCA M1-D & 5 & 2 & 1 & good \\
14 & MCA M1-P & 5 & 1 & 1 & \\
\hline
\end{tabular}

BA: indicates basilar artery, DSA: digital subtraction angiography, ICA-D: internal carotid artery distal, ICA-T: internal carotid artery terminal, MCA M1-P: middle cerebral artery M1 proximal, MCA M1-D: middle cerebral artery M1 distal, Collateral grades mean as bellows. A score of 1 was assigned if collaterals reconstituted the distal portion of the occluded vessel segment. A score of 2 was assigned if collaterals reconstituted vessels in the proximal portion of the segment adjacent to the occluded vessel. A score of 3 was assigned if collaterals reconstituted vessels in the distal portion of the segment adjacent to the occluded vessel. A score of 4 was assigned if collaterals reconstituted vessels two segments distal to the occluded vessel. A score of 5 was assigned if there was little or no significant reconstitution of the territory of the occluded vessel.

that would otherwise result from the inadequate device selection.

Several methods have been used to visualize the distal portions of the occluded vessel. Smit et al. reported imaging of collateral vessels using timing-invariant CT angiography (TI-CTA) constructed using CT-perfusion data. ${ }^{10)}$ TI-CTA was performed using a 128-detector-row CT scanner with $40 \mathrm{ml}$ of nonionic contrast medium. In that study, TI-CTA yielded more detailed information regarding collateral vessels when compared with standard CTA. This modality requires a CT scanner unit with reconstruction of images at a CT workstation, and operators have to wait at the CT unit until CTA images are processed. If patients are

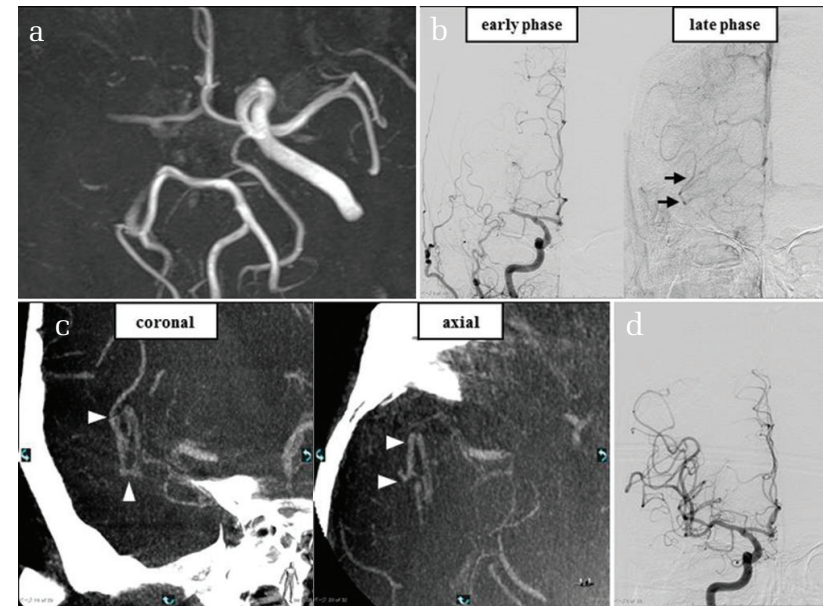

Fig. 1 A 78-year-old male presents with right ICA stenosis and right MCA M1 occlusion. (a) MRA shows right ICA and MCA M1 tandem occlusion. (b) Conventional DSA, AP view, early arterial phase and late arterial phase, demonstrate occlusion of the right MCA M1 with collateral leptomeningeal circulation and partial retrograde reconstitution of distal potion of MCA M2 (arrows). (c) Ao-CBCT coronal view and axial view demonstrate MCA M1 occluded site and reconstitution of the proximal potion of the MCA M2 (arrowheads). (d) Conventional DSA, AP view, after EVT with penumbra system, TICI $2 \mathrm{~b}$ recanalization was obtained.

to undergo EVT in the hyper-acute phase, a time delay from CTA examination to groin puncture (for EVT) may occur due to patient transfer time from the CT unit to the angiography suite. In contrast, Ao-CBCT images are acquired in the angiography suite, thereby obviating any time delay that would otherwise be associated with a transfer from the CT unit. Therefore, Ao-CBCT can shorten the time to EVT when compared with CTA assessment.

Another vessel evaluation method that can be conducted in the angiography suite is intravenously enhanced flat-detector CT (IV-FDCT). ${ }^{11)}$ IV-FDCT is obtained via propeller scan with contrast injection (total, $80 \mathrm{ml}$ ) via a pigtail catheter deployed in the inferior vena cava or right atrium. IV-CBCT can visualize the occluded site and the distal vessels. However, IV-CBCT requires more contrast medium than Ao-CBCT. Moreover, IV-CBCT requires two groin puncture sites: one site for EVT, and another site for contrast injection via the inferior vena cava or right atrium. This additional groin puncture may result in a time delay for recanalization or can result in puncture-related complications. Because Ao-CBCT can be performed via a single groin puncture site for EVT and the same catheter coaxial system for recanalization therapy, Ao-CBCT can shorten the time 


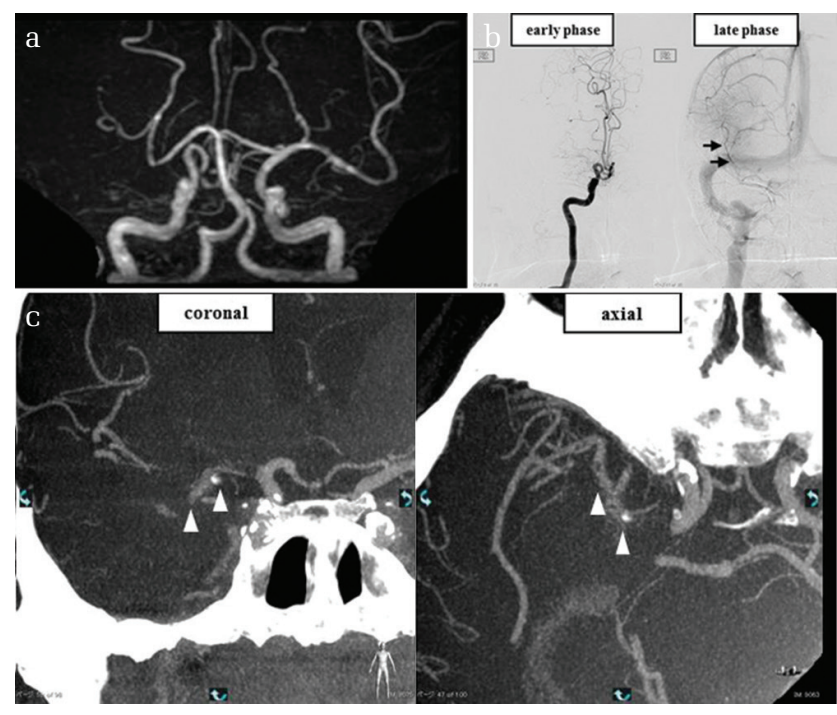

Fig. 2 An 80-year-old male with atrial fibrillation presented with right MCA M1 proximal occlusion. (a) MRA shows right MCA M1 proximal occlusion. (b) Conventional DSA, AP view, early arterial phase and late arterial phase, demonstrate occlusion of the right MCA M1 proximal with collateral leptomeningeal circulation and partial retrograde reconstitution of the distal potion of the MCA M2 (arrows). (c) Ao-CBCT coronal view and axial view demonstrate MCA M1 occluded site and reconstitution of the middle potion of the MCA M1 (arrowheads). During diagnostic angiography, his symptoms improved. We did not perform EVT.

from assessment to recanalization when compared to IV-CBCT.

Nagahata et al. described the use of basi-parallel anatomic scanning (BPAS) using MRI. ${ }^{13)}$ BPAS is a surface scanning MR imaging technique that characterizes the surface appearance of the intracranial VA-BA. Kato et al. described the use of Sylvian vallecula-parallel anatomic scanning-MR imaging, which can visualize the surface appearance of MCA. ${ }^{14)}$ Both of these methods can visualize the outer layer of vessels, even if the vessels are occluded. Moreover, these methods are not invasive and do not require contrast medium. While these methods can estimate the inner diameter of the occluded site and distal vessels of the occluded site, the estimated inner diameter may not reflect the actual diameter. For example, if the vessel occlusion occurred from atherosclerosis, the inner diameter might be overestimated. In this situation, the use of devices that require selection according to the estimated vessel diameter may be problematic and lead to vessel injury. On the other hand, Ao-CBCT can characterize the inner lumen just proximal to the occluded site and of the distal vessels. The acquired lumen diameter information can facilitate appropriate device selection for recanalization and reduce the complication risk that would otherwise be associated with suboptimal device selection.

Ao-CBCT may be able to shorten the time from vessel evaluation to recanalization. If the occluded site and collateral flow are assessed via conventional angiography, an injection of contrast from several arteries is needed. For example, in the case of ICA proximal occlusion, injections from the ipsilateral common carotid artery, contralateral ICA and VA are needed to assess the occlusion site and distal collateral flow. In comparison, Ao-CBCT can characterize the occluded site and distal portions at the same time. Therefore, Ao-CBCT requires only one target vessel angiography and can thereby shorten the time from a groin puncture to recanalization.

There are some limitations in this study. First, we did not compare Ao-CBCT and CTA images directly. In our hospital, patients with suspected stroke are initially assessed by MRI and MRA. CT is performed only for those who have contraindications to MRI or who have a hemorrhagic stroke defined by MRI. In this study, because all patients were initially assessed by MRI and MRA, CTA data were not available. If both examinations are performed in the acute phase, a time delay in treatment may occur, and there is a higher risk of subsequent renal dysfunction resulting from the repetitive use of contrast. Second, most of our patients had an occlusion site in the anterior circulation system. Only one of 14 patients suffered ischemic stroke related to occlusion of the posterior circulation system. Therefore, validation of these results using a larger number of patients with posterior circulation vessel occlusion is needed.

\section{Conclusions}

Ao-CBCT is an efficient method to obtain detailed information regarding occluded vessels in patients with ischemic stroke patients when compared with conventional examination methods. This modality requires a lower volume of contrast agent and a shorter time when compared with IV-CBCT and CTA, while yielded enough sufficient information to guide the selection of treatment strategy. Moreover, this modality may shorten the time to recanalization and obviates the need for contrast injection of unoccluded vessels when evaluating collaterals. 


\section{Conflicts of Interest Disclosure}

T.A has received honoraria from Philips Healthcare. Y.M has received honoraria from Terumo, Johnson \& Johnson, Covidien, Medico's Hirata, Stryker, Asahi Intecc, Kaneka and Philips Healthcare. Other authors have no actual or potential conflicts of interest. Authors who are members of the Japan neurosurgical society (JNS) have registered online Self-reported COI Disclosure Statement Forms through the website for JNS members.

\section{References}

1) Smith WS, Sung G, Saver J, Budzik R, Duckwiler G, Liebeskind DS, Lutsep HL, Rymer MM, Higashida RT, Starkman S, Gobin YP; Multi MERCI Investigators, Frei D, Grobelny T, Hellinger F, Huddle D, Kidwell C, Koroshetz W, Marks M, Nesbit G, Silverman IE: Mechanical thrombectomy for acute ischemic stroke: final results of the Multi MERCI trial. Stroke 39: 1205-1212, 2008

2) Bose A, Henkes H, Alfke K, Reith W, Mayer TE, Berlis A, Branca V, Sit SP; Penumbra Phase 1 Stroke Trial Investigators: The Penumbra System: a mechanical device for the treatment of acute stroke due to thromboembolism. AJNR Am J Neuroradiol 29: 1409-1413, 2008

3) Saver JL, Jahan R, Levy EI, Jovin TG, Baxter B, Nogueira RG, Clark W, Budzik R, Zaidat OO; SWIFT Trialists: Solitaire flow restoration device versus the Merci Retriever in patients with acute ischemic stroke (SWIFT): a randomized, parallelgroup, non-inferiority trial. Lancet 380: 1241-1249, 2012

4) Nogueira RG, Lutsep HL, Gupta R, Jovin TG, Albers GW, Walker GA, Liebeskind DS, Smith WS; TREVO 2 Trialists: Trevo versus Merci retrievers for thrombectomy revascularization of large vessel occlusion in acute ischemic stroke (TREVO 2): a randomized trial. Lancet 380: 1231-1240, 2012

5) Berkhemer OA, Fransen PS, Beumer D, van den Berg LA, Lingsma HF, Yoo AJ, Schonewille WJ, Vos JA, Nederkoorn PJ, Wermer MJ, van Walderveen MA, Staals J, Hofmeijer J, van Oostayen JA, Lycklama à Nijeholt GJ, Boiten J, Brouwer PA, Emmer BJ, de Bruijn SF, van Dijk LC, Kappelle LJ, Lo RH, van Dijk EJ, de Vries J, de Kort PL, van Rooij WJ, van den Berg JS, van Hasselt BA, Aerden LA, Dallinga RJ, Visser MC, Bot JC, Vroomen PC, Eshghi O, Schreuder TH, Heijboer RJ, Keizer K, Tielbeek AV, den Hertog HM, Gerrits DG, van den Berg-Vos RM, Karas GB, Steyerberg EW, Flach HZ, Marquering HA, Sprengers ME, Jenniskens SF, Beenen LF, van den Berg R, Koudstaal PJ, van Zwam WH, Roos YB, van der Lugt A, van Oostenbrugge RJ, Majoie CB, Dippel DW; MR CLEAN Investigators: A randomized trial of intraarterial treatment for acute ischemic stroke. $N$ Engl J Med 372: 11-20, 2015

6) Goyal M, Demchuk AM, Menon BK, Eesa M, Rempel JL, Thornton J, Roy D, Jovin TG, Willinsky RA, Sapkota BL, Dowlatshahi D, Frei DF, Kamal NR, Montanera WJ, Poppe AY, Ryckborst KJ, Silver FL, Shuaib A, Tampieri D, Williams D, Bang OY, Baxter BW, Burns PA, Choe H, Heo JH, Holmstedt CA, Jankowitz B, Kelly M, Linares G, Mandzia JL, Shankar J, Sohn SI, Swartz RH, Barber PA, Coutts SB, Smith EE, Morrish WF, Weill A, Subramaniam S, Mitha AP, Wong JH, Lowerison MW, Sajobi TT, Hill MD; ESCAPE Trial Investigators: Randomized assessment of rapid endovascular treatment of ischemic stroke. N Engl J Med 372: 1019-1030, 2015

7) Campbell BC, Mitchell PJ, Kleinig TJ, Dewey HM, Churilov L, Yassi N, Yan B, Dowling RJ, Parsons MW, Oxley TJ, Wu TY, Brooks M, Simpson MA, Miteff F, Levi CR, Krause M, Harrington TJ, Faulder KC, Steinfort BS, Priglinger M, Ang T, Scroop R, Barber PA, McGuinness B, Wijeratne T, Phan TG, Chong W, Chandra RV, Bladin CF, Badve M, Rice H, de Villiers L, Ma H, Desmond PM, Donnan GA, Davis SM; EXTEND-IA Investigators: Endovascular therapy for ischemic stroke with perfusion-imaging selection. $N$ Engl J Med 372: 1009-1018, 2015

8) Saver JL, Goyal M, Bonafe A, Diener HC, Levy EI, Pereira VM, Albers GW, Cognard C, Cohen DJ, Hacke W, Jansen O, Jovin TG, Mattle HP, Nogueira RG, Siddiqui AH, Yavagal DR, Baxter BW, Devlin TG, Lopes DK, Reddy VK, du Mesnil de Rochemont R, Singer OC, Jahan R; SWIFT PRIME Investigators: Stent-retriever thrombectomy after intravenous t-PA vs. t-PA alone in stroke. $N$ Engl J Med 372: 2285-2295, 2015

9) Jovin TG, Chamorro A, Cobo E, de Miquel MA, Molina CA, Rovira A, San Román L, Serena J, Abilleira S, Ribó M, Millán M, Urra X, Cardona P, López-Cancio E, Tomasello A, Castaño C, Blasco J, Aja L, Dorado L, Quesada H, Rubiera M, Hernandez-Pérez M, Goyal M, Demchuk AM, von Kummer R, Gallofré M, Dávalos A; REVASCAT Trial Investigators: Thrombectomy within 8 hours after symptom onset in ischemic stroke. $N$ Engl J Med 372: 2296-2306, 2015

10) Smit EJ, Vonken EJ, van Seeters T, Dankbaar JW, van der Schaaf IC, Kappelle LJ, van Ginneken B, Velthuis BK, Prokop M: Timing-invariant imaging of collateral vessels in acute ischemic stroke. Stroke 44: 2194-2199, 2013

11) Blanc R, Pistocchi S, Babic D, Bartolini B, Obadia $\mathrm{M}$, Alamowitch S, Piotin M: Intravenouus flatdetector CT angiography in acute ischemic stroke management. Neuroradiology 54: 383-391, 2012

12) Christoforidis GA, Mohammad Y, Kehagias D, Avutu B, Slivka AP: Angiographic assessment of pial collaterals as a prognostic indicator following 
intra-arterial thrombolysiis for acute ischemic stroke. AJNR Am J Neuroradiol 26: 1789-1797, 2005

13) Nagahata $M$, Abe $Y$, Ono $S$, Hosoya $T$, Uno $S$ : Surface appearance of the vertebrobasilar artery revealed on basiparallel anatomic scanning (BPAS)MR imaging: its role for brain MR examination. AJNR Am J Neuroradiol 26: 2508-2513, 2005

14) Kato Y, Dembo T, Takeda H, Uchino A, Deguchi I, Furuya D, Tanahashi N: Outer contour of middle cerebral artery revealed by sylvian vallecula-parallel anatomic scanning-magnetic resonance imaging: a method useful for detecting dissection. Arch Neurol 67: 1278-1279, 2010

Address reprint requests to: Yuji Matsumaru, MD, Department of Neuro-Endovascular Therapy, Toranomon Hospital, 2-2-2 Toranomon, Minato-ku, Tokyo 105-8470, Japan. e-mail: yujimatsumaru@me.com 